\title{
Resíduos agroindustriais para produção biotecnológica de xilitol ${ }^{1}$
}

\section{Agro-industrial residues in biotechnological production of xylitol}

\author{
Carolina Tamanini ${ }^{2}$ Maria Celia de Oliveira Hauly ${ }^{3 *}$
}

\begin{abstract}
Resumo
Resíduos lignocelulósicos tais como bagaço de cana-de-açúcar, palha de arroz, casca de aveia e resíduos florestais representam fontes abundantes e não dispendiosas de carboidratos (celulose e hemicelulose) com potencial aplicação em processos de conversão química ou microbiana em produtos de interesse comercial. Xilose, o açúcar predominante na fração hemicelulósica pode ser convertido em xilitol. O xilitol é um poliol com propriedades físico-química importantes para as indústrias alimentícia e farmacêutica. É um composto com doçura semelhante à da sacarose, anticariogênico, tolerado por diabéticos e recomendado para pessoas obesas. Este poliol é produzido ,em grande escala, por hidrogenação catalítica da xilose presente em hidrolisados lignocelulósicos, porém as soluções obtidas por este processo requerem onerosos passos de purificação para obtenção do xilitol puro. Alternativamente, pode ser produzido por métodos biotecnológicos utilizando leveduras e/ou enzimas. Tais processos consistem na fermentação de hidrolisados hemicelulósicos de resíduos agroindustriais, e podem competir com o tradicional processo químico. O presente trabalho teve como objetivo a realização de uma revisão bibliográfica sobre xilitol destacando os aspectos estruturais, as vias de obtenção e aplicações, principais substratos hemicelulósicos empregados em sua produção, hidrólise ácida e tratamento do hidrolisado hemicelulósico para utilização como substrato na obtenção de xilitol por via microbiana.

Palavras-chave: Resíduos lignocelulósicos, hidrólise ácida, hidrolisado hemicelulósico, tratamento do hidrolisado, fermentação, xilitol.
\end{abstract}

\begin{abstract}
Lignocellulosic residues, such as sugarcane bagasse, rice and oat straw and forest cuttings, are abundant and inexpensive sources of carbohydrates (cellulose and hemicellulose) with potential application in several conversion processes. Xylose, the predominant sugar in the hemicellulose fraction can be converted to xylitol. Xylitol is a polyol with some interesting properties that can make it an important product for the food and pharmaceutical industry. It is a compound with sweetness similar to that sucrose, is non-cariogenic, tolerated by diabetics and recommended for obese people. This polyol is currently produced by chemical catalysis of the xylose from lignocellulosic residues. However, this process needs expensive purification steps to obtain pure xylitol. Alternatively, it can be produced by biotechnological methods, using microorganisms, specially yeasts. These processes consist of hemicellulosic hydrolysate fermentation from agro-industrial residues, wich could compete with the traditional chemical method. The present work aims the accomplishment of a review about xylitol detaching
\end{abstract}

1 Parte da monografia apresentada ao Departamento de Bioquímica e Biotecnologia da Universidade Estadual de Londrina, em 2003, para obtenção do título de Especialista em Bioquímica Aplicada.

2 Bacharel em Química, Especialista em Bioquímica Aplicada e aluna do Programa de Pós-graduação em Biotecnologia (nível: mestrado), Universidade Estadual de Londrina.

3 Docente do Departamento de Bioquímica e Biotecnologia da Universidade Estadual de Londrina, Caixa Postal 6001, CEP 86051990, Londrina-PR, E-mail: mcoh@uel.br

* Autor para correspondência. 
the structural aspects, ways of attainment and applications; main hemicellulosic substrates used in its production; acid hydrolysis and treatment of the hemicellulosic hydrolysate for use as substrate to produce xylitol by microbial way.

Key words: Lignocellulosic residues, acid hydrolysis, hemicellulosic hydrolysate, hidrolysate treatment, fermentation, xylitol.

\section{Introdução}

O Brasil é conhecido por seu grande potencial de produção de recursos renováveis tais como produtos agrícolas, florestais e resíduos como o bagaço de cana-de-açúcar, a palha de arroz, a palha de trigo, a casca de aveia e as aparas de madeira. A produção de resíduos agroindustriais é de aproximadamente 250 milhões de toneladas/ano. A utilização adequada destes resíduos ajuda a minimizar problemas ambientais e energéticos, além disso, pode gerar produtos com relevantes aplicações na indústria farmacêutica e de alimentos (ROBERTO et al., 1995; SOUZA; SANTOS, 2002).

Estes resíduos agrícolas, em geral, contêm cerca de 20 a $60 \%$ de celulose, 20 a $30 \%$ de hemicelulose e 15 a 30\% de lignina. O bagaço de cana, farelo de trigo e de arroz, por exemplo, contém cerca de 25 a $40 \%$ de celulose, 20 a $35 \%$ de hemicelulose e 15 a $35 \%$ de lignina (OLIVEIRA; CARVALHO; SCHUWAN, 2003). Estes materiais podem ser utilizados como substratos para processos fermentativos pois mais de $70 \%$ da matéria seca é constituída de carboidratos. A maior parte é celulose, uma $\beta$ (1-4) glucana que pode ser convertida em glicose. As hemiceluloses são heteropolímeros de pentoses e hexoses e correspondem de 10 a $40 \%$ da matéria seca dos resíduos lignocelulósicos. Esta fração pode ser convertida em açúcares monoméricos, principalmente xilose, em temperaturas abaixo de $200^{\circ} \mathrm{C}$ na presença de ácidos diluídos (NEUREITER et al., 2002). A xilose pode ser diretamente convertida em xilitol por vias biotecnológicas utilizando leveduras (ROBERTO; MANCILHA; SATO, 1999).

O xilitol é um carboidrato natural com poder adoçante semelhante ao da sacarose. Apresenta propriedades anticariogênicas e seu metabolismo em humanos é independente de insulina. Este poliol é produzido em larga escala por hidrogenação catalítica de soluções ricas em xilose, um processo oneroso pelo número e tipos de etapas de purificação requeridas. A via biotecnológica apresenta-se como alternativa à via química de obtenção de xilitol em função do alto custo do processo químico (PARAJÓ; DOMÍNGUEZ; DOMÍNGUEZ; 1998a) e da capacidade de microrganismos fermentarem a xilose presente nos hidrolisados. O metabolismo de xilose em leveduras consiste em sua redução a xilitol através da enzima xilose redutase que requer como cofator o NADPH $+\mathrm{H}^{+}$, seguida da oxidação à xilulose pela enzima xilitol desidrogenase que requer como cofator o NAD ${ }^{+}$(FELIPE et al., 2003).

Um aspecto interessante na produção biotecnológica de xilitol é o fato de que durante o processo de hidrólise ácida do resíduo agroindustrial, além da liberação dos açúcares, é comum ocorrer a formação de compostos tóxicos provenientes da degradação de hexoses e pentoses, como 5hidroximetilfurfural e furfural, respectivamente ou da lignina, como compostos aromáticos, fenólicos e aldeídicos. Também é comum a liberação de substâncias da própria estrutura lignocelulósica como o ácido acético ou dos equipamentos de hidrólise como íons ferro, cromo, níquel e cobre (MUSSATO; ROBERTO, 2002). Estes compostos, quando presentes no hidrolisado, são inibidores potenciais do metabolismo microbiano (ALVES et al., 1998). O efeito inibitório pode ser superado submetendo-se o hidrolisado a tratamentos adequados que incluem alterações do $\mathrm{pH}$ do meio com adição de ácidos e bases e utilização de carvão ativo.

Esta revisão bibliográfica sobre xilitol tem como objetivo destacar os aspectos estruturais, as vias de obtenção e aplicações, principais substratos hemicelulósicos empregados em sua produção, 
hidrólise ácida e tratamento do hidrolisado hemicelulósico para utilização como substrato na obtenção de xilitol por via microbiana.

\section{Revisão Bibliográfica}

\section{Propriedades físico-químicas do xilitol}

O xilitol é um poliálcool de cinco carbonos que pode ser encontrado na natureza em muitas frutas e vegetais tais como alface, couve flor, ameixas, framboesas, morangos, uvas, bananas, assim como em leveduras, liquens e cogumelos porém, em quantidades inferiores a $0,9 \mathrm{~g} / 100 \mathrm{~g}$ (PARAJÓ; DOMÍNGUEZ; DOMÍNGUEZ, 1998a).

Sua fórmula empírica é $\mathrm{C}_{5} \mathrm{H}_{12} \mathrm{O}_{5}$ e sua massa molecular 152,15 g.mol-1. Trata-se de um pó branco, cristalino, sem odor, altamente solúvel em água (64,2 g/ $100 \mathrm{~mL}$ ) e apresenta ponto de fusão na faixa de 93,4 a 94,7 C (AGUIAR; OETTERER; MENEZES, 1999).

O xilitol é um intermediário do metabolismo de carboidratos em mamíferos. Um homem adulto produz de 5 a $15 \mathrm{~g}$ deste poliálcool por dia (PARAJÓ; DOMÍNGUEZ; DOMÍNGUEZ, 1998a). O fato de ser lentamente absorvido no trato gastrintestinal e de seu metabolismo ser independente da insulina possibilita sua utilização como adoçante para diabéticos (WINKELHAUSEN; KUSMANOVA, 1998). O poder edulcorante é de 0,8 a 1,1 vezes a da sacarose e valor calórico de $2,4 \mathrm{kcal} / \mathrm{g}$. Este poliol é 2,4 vezes mais doce do que o manitol e 2 vezes mais que o sorbitol. Entretanto, o poder adoçante pode variar com o pH, concentração de sais e temperatura. Apresenta ainda uma agradável sensação refrescante devido ao valor negativo do calor específico de dissolução ( $-34,8 \mathrm{cal} / \mathrm{g})$ (AGUIAR; OETTERER; MENEZES, 1999). Outra propriedade importante do xilitol é o fato de não ser fermentado pela maioria dos microrganismos da cavidade bucal humana exercendo efeito anticariogênico e inibindo a desmineralização do esmalte dos dentes (BIRKHED, 1994).

\section{Aplicações do Xilitol}

Pode ser empregado isoladamente ou em associação com outros adoçantes em produtos de panificação, geléias, marmeladas, gelatinas, sobremesas, gomas de mascar, refrigerantes e sorvetes. Também pode ser utilizado em produtos farmacêuticos e de higiene oral (WINKELHAUSEN; KUSMANOVA, 1998). O uso mais significante, entretanto, é na substituição do açúcar presente em produtos altamente açucarados, como o leite condensado, que podem caramelizar durante o processamento ou a estocagem. A reação de Maillard, ou seja, reação química entre açúcares e proteínas acelerada por altas temperaturas e que provoca escurecimento, não ocorre com o xilitol pois este açúcar não têm grupos aldeídicos nem cetônicos (AGUIAR; OETTERER; MENEZES, 1999).

Também pode ser utilizado na prevenção ou tratamento de doenças como diabetes, obesidade, insuficiência da enzima glicose 6-fosfatodesidrogenase, otite, osteoporose e fibrose cística (RODRIGUES et al., 2003a).

\section{Obtenção do xilitol}

A obtenção de xilitol pode ser realizada de 3 maneiras: extração líquido-sólido, redução química da xilose e conversão biotecnológica de soluções contendo xilose (DOMÍNGUEZ et al., 1999). Tanto o processo químico quanto o biotecnológico se iniciam com soluções de xilose oriundas da hidrólise de materiais lignocelulósicos ricos em xilanas (CRUZ et al., 2000).

- Extração líquido-sólido: o xilitol presente em frutas e vegetais bem como em leveduras, liquens, algas e cogumelos pode ser recuperado por extração líquidosólido, mas como ocorre em baixas quantidades, menos que $0,900 \mathrm{~g} / 100 \mathrm{~g}$, este processo se torna economicamente inviável (PARAJÓ; DOMÍNGUEZ; DOMÍNGUEZ, 1998a).

- Redução química da xilose: a produção de xilitol em escala comercial é realizada por um processo 
químico patenteado em 1977 por Asko Melaja e Lauri Hamalainen (LIMA; BERLINCK, 2003). Este processo inclui quatro etapas básicas: 1. Obtenção da xilose por hidrólise ácida de material vegetal rico em xilana; 2 . Purificação da xilose obtida; 3. Hidrogenação catalítica da xilose formando xilitol e 4. Cristalização do composto. O rendimento do processo químico e a qualidade do xilitol dependem da pureza da solução inicial de xilose, já que a presença de impurezas interfere na reação catalítica. São necessárias operações de purificação (como troca iônica, descoloração e fracionamento cromatográfico) para obtenção de uma solução de xilose de elevada pureza. Após a remoção do catalisador por filtração e troca iônica, a solução de xilitol é concentrada, fracionada por cromatografia e cristalizada para obtenção do produto puro. As etapas de purificação aumentam o tempo do processo e encarecem o produto (LIMA; BERLINCK, 2003).

- Conversão biotecnológica de soluções contendo xilose: o xilitol pode ser produzido biotecnologicamente utilizando leveduras e/ou enzimas. Este processo consiste na fermentação de hidrolisados hemicelulósicos obtidos de resíduos agroindustriais e pode competir com o tradicional processo químico (FAVERI et al., 2003). O xilitol é um intermediário do metabolismo de D-xilose em leveduras, como pode ser observado na figura 1 onde é representado um esquema simplificado do metabolismo desta pentose nestes microrganismos.

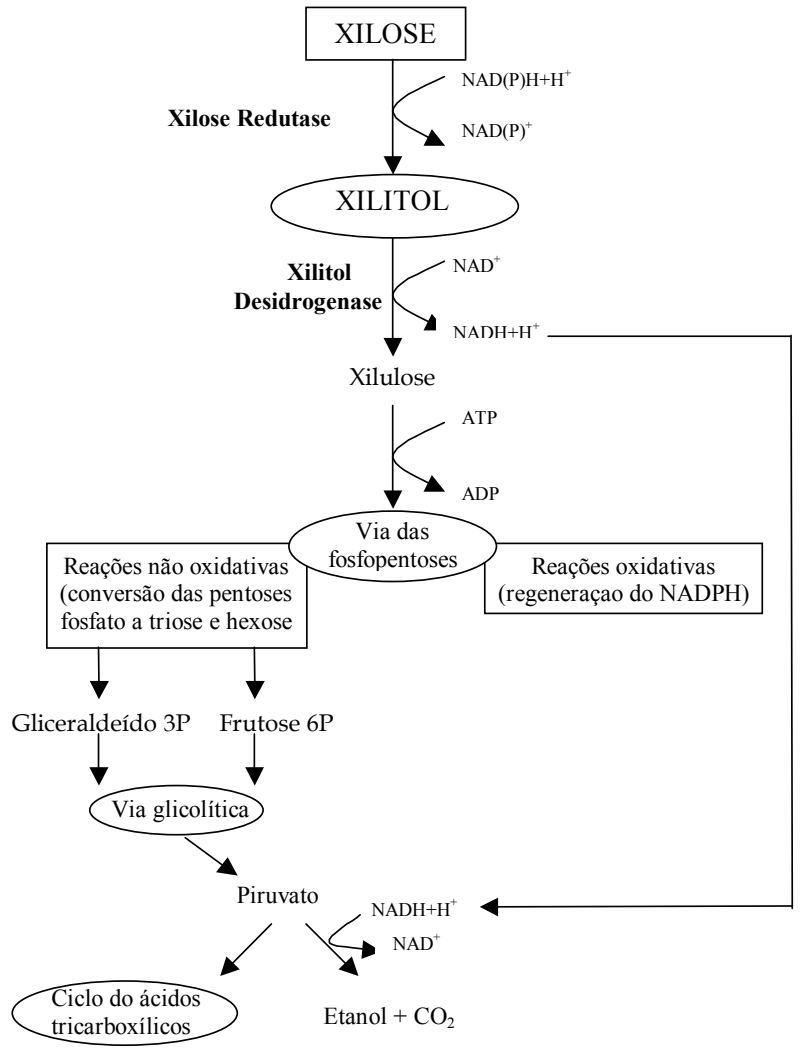

Figura 1. Esquema simplificado do metabolismo de xilose em leveduras.

Fonte: Parajó, Domínguez e Domïnguez (1998a). Adaptado.

A xilose redutase é NADPH dependente e xilitol desidrogenase é $\mathrm{NAD}^{+}$dependente. A regeneração do NADPH ocorre na via das pentoses fosfato e a regeneração do $\mathrm{NAD}^{+}$ocorre somente na cadeia respiratória com o oxigênio como aceptor final de elétrons. Porém, sob suprimento limitado de oxigênio, não ocorre a completa reoxidação do cofator, levando a um desequilíbrio redox. Uma baixa concentração de $\mathrm{NAD}^{+}$diminui a taxa de oxidação do xilitol mas não afeta sua produção na mesma extensão, induzindo sua excreção no meio de cultivo. Um alto grau de aeração favorece o crescimento celular, prejudicando o acúmulo do xilitol (AGUIAR JR et al., 2002). A fosforilação da D-xilulose a D-xilulose-5-fosfato é catalisada pela enzima xilulose quinase e envolve gasto de energia na forma de ATP. A D-xilulose-5fosfato formada pode ser convertida a piruvato através da conexão com a via das pentoses fosfato. 
O piruvato obtido pode ser descarboxilado a acetaldeido e reduzido a etanol ou a acetil-CoA pode entrar no ciclo dos ácidos tricarboxílicos que acoplado à cadeia de transporte de elétrons, regenera $\mathrm{NAD}^{+}$ e ATP (MUSSATO; ROBERTO, 2002).

\section{Resíduos lignocelulósicos}

A utilização de resíduos provenientes da exploração da biomassa lignocelulósica para obtenção de bioprodutos é uma alternativa para a produção de energia e de alimentos pois a madeira e os resíduos da agroindústria constituem reservas naturais renováveis disponíveis em grandes quantidades (ALVES, 2001). Os materiais lignocelulósicos incluem vários resíduos agrícolas (palhas, cascas, cavacos), madeiras duras provenientes de árvores de folhas decíduas (dicotiledôneas), madeiras moles provenientes de coníferas e resíduos das indústrias de papel. A composição destes materiais é bastante variável. O maior componente é a celulose (35-50\%) seguida pela hemicelulose (20-35\%) e lignina (1025\%) (SAHA, 2003).

A celulose é um homopolissacarídeo linear formado de unidades de glicose unidas por ligações glicosídicas do tipo $\beta(1-4)$. As cadeias de celulose agregam-se formando fibrilas e apresentam pontes de hidrogênio entre os grupamentos hidroxila intra e intercadeias, o que resulta na cristalinidade da celulose. Essas regiões cristalinas, nas quais as cadeias estão ordenadas paralelamente, são separadas por regiões menos ordenadas, conhecidas como amorfas (GALDEANO, 2001) enquanto as hemiceluloses são compostas pelos açúcares glicose, manose e galactose (hexoses) e xilose e arabinose (pentoses), podendo ainda apresentar quantidades variáveis de ácidos urônicos, grupos acetila e desoxihexoses em alguns tipos de madeira. Esses açúcares apresentam-se na forma de polímeros ramificados, de menor massa molecular que a celulose e podem ser homoplímeros ou heteropolímeros (FERRAZ, 2001). A lignina é um polímero vegetal derivado dos álcoois hidroxicinamílicos p-coumarílico, coniferílico e sinapílico, também chamados de monolignóis (ENDT et al., 2000) e atua como ligante das fibras de celulose, contribuindo para a resistência e rigidez do tecido da madeira (SHREVE; BRINCK JR, 1977). Existe ainda uma fração menor da madeira, formada basicamente por compostos fenólicos e resinas, que comumente são chamados de extrativos (solúveis em solventes orgânicos e água) e compreendem cerca de 2 a 4\% (FERRAZ, 2001).

A Tabela 1 mostra a composição química de alguns resíduos lignocelulósicos.

Tabela 1. Composição química parcial de alguns resíduos lignocelulósicos.

\begin{tabular}{lllll}
\hline \multicolumn{1}{c}{$\begin{array}{c}\text { Resíduos } \\
\text { Lignocelulósicos }\end{array}$} & \multicolumn{1}{c}{$\begin{array}{c}\text { Celulose } \\
(\%)\end{array}$} & $\begin{array}{c}\text { Hemicelulose } \\
(\mathbf{\%})\end{array}$ & $\begin{array}{c}\text { Lignina } \\
\mathbf{( \% )}\end{array}$ & \multicolumn{1}{c}{ Referência } \\
\hline Farelo de cevada & 23,0 & 32,7 & 24,4 & Cruz et al., 2000 \\
Sabugo de milho & 31,7 & 34,7 & 20,3 & Cruz et al., 2000 \\
Folhas de milho & 37,6 & 34,5 & 12,6 & Cruz et al., 2000 \\
Eucalyptus globulus & 46,3 & 17,1 & 22,9 & Cruz et al., 2000 \\
Bagaço de cana & 40,19 & 26,42 & 25,15 & Neureiter et al., 2002 \\
Palha de arroz & 43,5 & 22 & 17,2 & Mussato; Roberto, 2002. \\
Palha de trigo & 33,81 & 31,83 & 20,12 & Cândido; Canilha, Silva, 2002 \\
Casca de aveia & 30,51 & 28,63 & 23,09 & Felipe et al., 2003 \\
Eucalyptus grandis & 40,20 & 15,67 & 26,9 & Canettieri; Silva; Carvalho Jr, 2003 \\
Palha de sorgo & 34 & 44 & 20 & Herrera et al., 2004 \\
\hline
\end{tabular}


A população humana produz milhões de toneladas de resíduos agroindustriais anualmente. A América Latina, por exemplo, produz aproximadamente 500 milhões de toneladas/ano de subprodutos agroindustriais, sendo que o Brasil produz quase a metade deste montante (SOUZA; SANTOS, 2002). Na maioria das vezes, esses rejeitos são dispensados no meio ambiente, provocando um acúmulo excessivo de matéria orgânica. Embora este tipo de poluente seja biodegradável, torna-se necessário um tempo mínimo para ser mineralizado e, em virtude da intensa atividade humana na terra, observa-se a cada dia um aumento na dificuldade de reciclagem natural desses nutrientes (BÔAS; ESPOSITO, 2000). Estes resíduos podem ser convertidos em energia, produtos químicos e alimentos. Entretanto, devido à sua natureza polissacarídica, não são diretamente utilizados pelos microrganismos de interesse industrial, sendo necessário hidrolisá-los para os respectivos monômeros (GARCIA; PRATA, 1998).

\section{Hidrólise ácida de materiais lignocelulósicos}

Ácidos diluídos podem ser utilizados para hidrólise parcial de materiais lignocelulósicos. Este procedimento, chamado pré-hidrólise, consiste na hidrólise da fração hemicelulósica, que é mais susceptível ao tratamento ácido, sendo que as frações de celulose e lignina permanecem inalteradas (AGUILAR et al., 2002) podendo ser utilizadas para a produção de soluções de glicose ou na fabricação de polpa de papel (HERRERA et al., 2001). Ácidos tais como sulfúrico, clorídrico e acético são comumente empregados como catalisadores nestes processos.

De acordo com Sun e Cheng (2002) há dois tipos de tratamentos de hidrólise com ácidos diluídos: alta temperatura (maior que $160^{\circ} \mathrm{C}$ ), processo contínuo e baixa carga de sólidos (5 a 10\% massa de substrato/ massa da mistura reacional) e baixa temperatura (menor que $160^{\circ} \mathrm{C}$ ) processo em batelada e alta carga de sólidos (10 a 40\%). Estes tratamentos permitem alcançar elevados rendimentos sendo que em temperaturas elevadas há favorecimento da hidrólise da celulose enquanto que sob condições mais brandas há maior conversão de xilanas em xilose.

Os ácidos utilizados como catalisadores nos processo de hidrólise liberam prótons que atuam nas ligações glicosídicas entre os monômeros de açúcar nas cadeias poliméricas. O rompimento destas ligações libera uma série de compostos, principalmente açúcares como xilose, glicose e arabinose (AGUILAR et al., 2002). São liberados também produtos indesejáveis para o processo fermentativo tais como furfural, proveniente da degradação de pentoses e 5-hidroximetilfurfural (5HMF) oriundo da desidratação de hexoses, havendo ainda formação de ácido fórmico pela degradação de compostos derivados do furano (furfural ou 5HMF) e de ácido levulínico produzido a partir da degradação de 5-HMF (PALMQVIST; HAHNHÄGERDAL, 2000). São gerados ainda ácido acético proveniente dos grupos acetil, compostos não estruturais correspondentes à fração extrativa e produtos de degradação da lignina (CRUZ et al., 2000) (fenóis e outros compostos aromáticos) e metais pesados como cromo, cobre, ferro e níquel provenientes da corrosão dos equipamentos de hidrólise (RODRIGUES et al., 2001). Uma vez presentes no hidrolisado, estes compostos são inibidores potenciais do metabolismo microbiano (ALVES et al., 1998), pela inibição das enzimas xilose redutase e xilitol desidrogenase (ALVES et al., 2002), limitando o consumo da fonte de carbono e interferindo na cinética de crescimento (MORITA; SILVA; MAUGERI, 2003).

\section{Mecanismo de hidrólise ácida de materiais lignocelulósicos}

De acordo com Herrera et al. (2001) as reações de hidrólise dos polímeros de açúcares com ácidos diluídos são bastante complexas uma vez que o substrato está em fase sólida e o catalisador em fase líquida.

O mecanismo de hidrólise consiste em: 
1. difusão dos prótons através da matriz lignocelulósica úmida;

2. protonação do oxigênio da ligação éter entre os monômeros de açúcar;

3. quebra da ligação éter e geração de um carbocátion como intermediário;

4. solvatação do carbocátion com água;

5. regeneração do próton e cogeração do monômero de açúcar, oligômero ou polímero, dependendo da posição da ligação éter rompida;

6. difusão dos produtos na fase líquida (se for permitido por sua forma e tamanho);

7. reinício do processo a partir do passo 2.

Tratamento do hidrolisado ácido para ser utilizado na bioconversão de xilitol

Vários tratamentos físico-químicos têm sido utilizados com o objetivo de minimizar o efeito tóxico dos hidrolisados sobre a fermentação tanto por transformação dos inibidores em compostos inativos quanto pela sua remoção (PARAJÓ; DOMÍNGUEZ; DOMÍNGEZ, 1998b). Dentre os diversos tratamentos utilizados para minimizar a concentração dos inibidores, a neutralização, a adsorção com carvão ativo e o uso de resinas de troca iônica são os mais empregados (MORITA; SILVA; MAUGERI, 2003).

De acordo com van Zyl (1988 apud MARTON, 2002) a elevação do $\mathrm{pH}$ do hidrolisado inicialmente ácido a valores maiores que 7,0 utilizando álcalis, resulta na precipitação de componentes tóxicos como íons de metais pesados, acetatos, taninos e terpenos. Este precipitado é então removido por centrifugação e o pH do meio é ajustado até que se obtenha uma condição de $\mathrm{pH}$ adequada para a atividade microbiana no processo fermentativo (ROBERTO; MANCILHA; SATO, 1999; FELIPE et al., 2003).

O tratamento com carvão ativo se fundamenta na capacidade deste material poroso, de origem natural, de adsorver sobre sua superfície diferentes tipos de moléculas através de forças fracas denominadas de Van der Waals. Estas forças são resultantes de uma atração intermolecular de tal modo que seu potencial é, basicamente, uma função da área superficial do material que varia de 600-1600 $\mathrm{m}^{2}$ dependendo da matéria prima empregada para sua fabricação (COSIDINE, 1974 apud ALVES, 2001).

Alves et al. (1998) utilizaram um planejamento fatorial de $2^{5-1}$ para avaliar as condições de tratamento do hidrolisado de bagaço de cana-de-açúcar para remoção dos compostos tóxicos assim como o tempo de fermentação. Foram verificadas a influência da base $\left(\mathrm{CaO}\right.$ ou $\left.\mathrm{Ca}(\mathrm{OH})_{2}\right)$, do ácido $\left(\mathrm{H}_{2} \mathrm{SO}_{4}\right.$ ou $\left.\mathrm{H}_{3} \mathrm{PO}_{4}\right)$, do $\mathrm{pH}$ final do tratamento básico $(7,0 \mathrm{ou}$ $10,0)$, da utilização ou não de carvão ativo $3 \%(\mathrm{p} / \mathrm{v})$ e do tempo de fermentação (45 ou 63 h). Depois de feitas as análises estatísticas, os fatores com efeitos significativos foram utilizados para experimentos adicionais com auxílio de uma modelagem estatística de $2^{2}$ para obtenção de um modelo matemático representativo desse processo fermentativo. $\mathrm{O}$ maior rendimento na bioconversão de xilose em xilitol $(0,79 \mathrm{~g} / \mathrm{g})$ foi obtido quando o $\mathrm{pH}$ do hidrolisado foi alterado de 0,5 para 7,0 com $\mathrm{CaO}$ e depois reduzido para 5,5 com $\mathrm{H}_{3} \mathrm{PO}_{4}$ e em seguida tratado com $2,4 \%$ de carvão ativo a $200 \mathrm{rpm}$, por $1 \mathrm{~h} \mathrm{a} 30^{\circ} \mathrm{C}$ e correspondeu à uma produtividade de $0,47 \mathrm{~g} / \mathrm{L} . \mathrm{h}$ depois de $63 \mathrm{~h}$ de fermentação utilizando a levedura Candida guilliermondii.

O processo de evaporação à vácuo do hidrolisado à baixa pressão com o objetivo de aumentar a concentração de xilose no meio reduz as concentrações de compostos tóxicos voláteis como ácido acético e furfural (RODRIGUES et al., 2003b). Morita, Silva e Maugeri (2003) verificaram redução de $56,5 \%$ de ácido acético, $81,79 \%$ de fenóis e de mais de $90 \%$ dos aldeídos furanos no hidrolisado de bagaço de cana-de-açúcar tratado com carvão ativo e concentrado à vácuo em relação ao hidrolisado original.

A utilização de carvão ativo apresenta a desvantagem de provocar uma perda considerável de xilose. Este problema pode ser superado realizando a neutralização e/ou purificação de hidrolisados hemicelulósicos com resinas de troca iônica que, além 
de evitarem a perda de xilose, removem eficientemente a cor, compostos fenólicos, ácidos orgânicos e inorgânicos, compostos furânicos e metais (NÁPOLES et al., 1998 apud VERDE, 2001).

\section{Obtenção de xilitol a partir de diferentes resíduos lignocelulósicos}

Cruz et al. (2000) utilizaram três resíduos agrícolas com alto conteúdo de xilanas (farelo de cevada, sabugos e folhas de milho) que foram hidrolisados em autoclave com soluções diluídas de ácido sulfúrico sob uma variedade de condições operacionais com o objetivo de obter soluções de xilose apropriadas para serem utilizadas como meio de fermentação para Debaryomyces hansenii.

As condições de hidrólise foram selecionadas com base no modelo cinético de geração de xilose e na quantidade de subprodutos tóxicos formados. Os hidrolisados foram suplementados com nutrientes e utilizados como meio de cultivo sem nenhum tratamento prévio de destoxificação. Os resultados obtidos com os três resíduos em estudo foram comparados com os resultados de Eucalyptus globulus, um resíduo muito utilizado em processo de pré-hidrólise que já tinha suas condições de hidrólise estabelecidas ( $3 \%$ de $\mathrm{H}_{2} \mathrm{SO}_{4}, 130^{\circ} \mathrm{C}$, relação madeira: solução ácida $1: 8$ e tempo de reação de $1 \mathrm{~h}$ ).

Os hidrolisados obtidos foram neutralizados com $\mathrm{CaCO}_{3}$ até pH 6,5 e o precipitado de $\mathrm{CaSO}_{4}$ foi removido por filtração sendo os sobrenadantes clarificados e, depois de suplementados com nutrientes e esterilizados, foram utilizados como meio de fermentação.

A Tabela 2 apresenta as condições ótimas de hidrólise assim como a composição dos hidrolisados antes da neutralização (A), após concentração por evaporação e esterilização (B) e depois de terminada a fermentação (C). A Tabela 3 traz os parâmetros fermentativos obtidos na fermentação dos hidrolisados de farelo de cevada, sabugos de milho, folhas de milho e madeira de eucalipto.
Foi observado que nos hidrolisados de farelo de cevada, sabugos de milho e folhas de milho, $95 \%$ da xilose havia sido consumida depois de $81 \mathrm{~h}$ de fermentação enquanto no hidrolisado de madeira de E. globulus o consumo foi de $80 \%$. A produção de xilitol foi fortemente inibida no meio de hidrolisado de madeira de E. globulus cuja concentração foi de apenas 1,5 g/L apresentando baixos valores de parâmetros fermentativos. Por outro lado, a produção de xilitol foi satisfatória nos meios constituídos dos hidrolisados de farelo de cevada, sabugos de milho e folhas de milho. Depois de $81 \mathrm{~h}$, as concentrações de xilitol variaram entre 21,2 a $29 \mathrm{~g} / \mathrm{L}$ sendo os valores de parâmetros fermentativos razoáveis, considerando-se a ausência de tratamentos de destoxificação dos hidrolisados.

Canilha (2001) estudou a influência da temperatura, concentração ácida e relação massa seca: solução ácida na hidrólise da palha de trigo mediante um planejamento fatorial para identificar os fatores importantes para o processo. A variável analisada foi a concentração de xilose presente no hidrolisado, que foi mais elevada quando a palha de trigo foi tratada com $1 \%$ de solução ácida, na proporção de 1:15 de palha seca: solução ácida, temperatura de $140^{\circ} \mathrm{C}$ e tempo de 20 minutos.

Canilha, Cândido e Silva (2002) utilizaram o hidrolisado hemicelulósico de palha de trigo concentrado, tratado, autoclavado a $111^{\circ} \mathrm{C}$ por 15 minutos e suplementado com $1 \mathrm{~g} / \mathrm{L}$ de sulfato de amônio e $5 \mathrm{~g} / \mathrm{L}$ de extrato de arroz como meio de cultivo para Candida guilliermondii para a produção de xilitol. A fermentação foi conduzida em biorreator utilizando inóculo de $0,5 \mathrm{~g}$ de células/L, a $30^{\circ} \mathrm{C}$, com agitação de $300 \mathrm{rpm}$ e aeração de 0,4 vvm. Amostras foram retiradas periodicamente para monitorar o consumo de xilose e produção de xilitol. A maior eficiência de conversão (96\%) foi obtida após 54 h de fermentação e apresentou os seguintes parâmetros fermentativos: produtividade de 0,503 g/L.h, rendimento de $0,889 \mathrm{~g}$ de xilitol/ $\mathrm{g}$ de xilose e produção de xilitol de 27,86 g/L. 
Tabela 2. Condições ótimas de hidrólise e composição química parcial dos hidrolisads de farelo de cevada, sabugos de milho, folhas de milho e madeira de eucalipto.

\begin{tabular}{|c|c|c|c|c|}
\hline Resíduo & $\begin{array}{l}\text { Condições de } \\
\text { hidrólise }\end{array}$ & $\underset{(\mathrm{g} / \mathrm{L})}{\mathrm{A}}$ & $\begin{array}{c}\mathrm{B} \\
(\mathrm{g} / \mathrm{L})\end{array}$ & $\underset{(g / L)}{C}$ \\
\hline \multirow[t]{7}{*}{ Farelo de cevada } & $\mathrm{H}_{2} \mathrm{SO}_{4} 3 \%$ & xilose: 35,6 & xilose 45,4 & xilose: 1,1 \\
\hline & 15 minutos & glicose: 5,9 & glicose: 7,5 & glicose: 0,3 \\
\hline & $1: 8$ & arabinose: 7,1 & arabinose: 9,1 & arabinose: 2,2 \\
\hline & & ac.acético: 2,4 & ac.acético: 3,1 & ac.acético: 0,5 \\
\hline & & furfural: 0,3 & furfural: 0,1 & furfural : $<0,1$ \\
\hline & & HMF: $<0,1$ & HMF: $<0,1$ & HMF: $<0,1$ \\
\hline & & & & $\begin{array}{r}\text { xilitol: } 26,6 \\
\text { etanol: } 6,7\end{array}$ \\
\hline \multirow[t]{7}{*}{ Sabugos de milho } & $\mathrm{H}_{2} \mathrm{SO}_{4} 2 \%$ & xilose: 35,3 & xilose: 42,9 & xilose: 2,2 \\
\hline & 15 minutos & glicose: 3,2 & glicose: 3,9 & glicose: 0,7 \\
\hline & $1: 8$ & arabinose: 4,6 & arabinose: 5,6 & arabinose: 4,7 \\
\hline & & ac.acético: 3,7 & ac.acético: 4,5 & ac.acético: 2,0 \\
\hline & & furfural: 0,3 & furfural: 0,2 & furfural: $<0,2$ \\
\hline & & HMF: $<0,1$ & HMF: $<0,1$ & HMF: $<0,1$ \\
\hline & & & & $\begin{array}{r}\text { xilitol: } 21,2 \\
\text { etanol: } 2,0\end{array}$ \\
\hline \multirow{7}{*}{ Folhas de milho } & $\mathrm{H}_{2} \mathrm{SO}_{4} 3 \%$ & xilose: 22,5 & xilose: 48,1 & xilose: 2,6 \\
\hline & 15 minutos & glicose: 2,0 & glicose: 4,3 & glicose: 0,5 \\
\hline & $1: 16$ & arabinose: 3,6 & arabinose: 7,7 & arabinose: 5,8 \\
\hline & & ac.acético: 2,3 & ac.acético: 4,6 & ac.acético: 1,6 \\
\hline & & furfural: 0,3 & furfural: 0,1 & furfural: $<0,1$ \\
\hline & & HMF: $<0,1$ & HMF: $<0,1$ & HMF: $<0,1$ \\
\hline & & & & $\begin{array}{r}\text { xilitol: } 21,2 \\
\text { etanol: } 5,4\end{array}$ \\
\hline \multirow{7}{*}{$\begin{array}{l}\text { Madeira de } E \text {. } \\
\text { globulus }\end{array}$} & $\mathrm{H}_{2} \mathrm{SO}_{4} 3 \%$ & xilose: 17,1 & xilose: 41,9 & xilose: 3,5 \\
\hline & 60 minutos & glicose: 1,7 & glicose: 3,2 & glicose: 2,1 \\
\hline & $1: 8$ & arabinose: 2,1 & arabinose: 5,1 & arabinose: 1,5 \\
\hline & & ac.acético: 5,2 & ac.acético: 12,6 & ac.acético: 12,3 \\
\hline & & furfural: 0,5 & furfural: 0,1 & furfural: $<0,1$ \\
\hline & & HMF: $<0,1$ & HMF: $<0,1$ & HMF: $<0,1$ \\
\hline & & & & $\begin{array}{l}\text { xilitol: } 1,5 \\
\text { etanol: } 9,7\end{array}$ \\
\hline
\end{tabular}

Composição dos hidrolisados antes da neutralização (A), após concentração por evaporação e esterilização (B) e depois de terminada a fermentação $(\mathrm{C})$.

Fonte: Cruz et al. (2000).

Tabela 3. Parâmetros fermentativos obtidos na fermentação dos hidrolisados de farelo de cevada, sabugos de milho, folhas de milho e madeira de eucalipto por Debaryomyces hansenii.

\begin{tabular}{lcccc}
\hline \multicolumn{1}{c}{ Resíduo } & $\mathbf{P}(\mathbf{g} / \mathbf{L})$ & $\mathbf{Q}_{\mathbf{p}}(\mathbf{g} / \mathbf{L} \cdot \mathbf{h})$ & $\mathbf{Y}_{\mathbf{P} / \mathbf{S}}(\mathbf{g} / \mathbf{g})$ & $\mathbf{q}_{\mathbf{p}}(\mathbf{g} / \mathbf{g . h})$ \\
\hline Farelo de cevada & 26,6 & 0,33 & 0,61 & 0,032 \\
Sabugos de milho & 21,2 & 0,26 & 0,52 & 0,025 \\
Folhas de milho & 29,0 & 0,36 & 0,64 & 0,036 \\
Madeira de E. globulus & 1,5 & 0,018 & 0,044 & 0,006 \\
\hline
\end{tabular}

P: produção máxima de xilitol; Qp: produtividade; $\mathrm{Y}_{\mathrm{P} / \mathrm{S}}$ : rendimento em produto; $\mathrm{q}_{\mathrm{p}}$ : taxa de formação de produto específico Fonte: Cruz et al. (2000). 
Canettieri, Silva e Felipe (2001) avaliaram a bioconversão de xilose em xilitol por Candida guilliermondii a partir de hidrolisado hemicelulósico de Eucalyptus grandis obtido por hidrólise com ácido sulfúrico. O hidrolisado foi obtido de cavacos de eucaliptos com dimensões de 20 x 10 x $5 \mathrm{~mm}$ com $29 \%$ de umidade. A relação (p/v) cavacos: solução de $\mathrm{H}_{2} \mathrm{SO}_{4} 0,35 \%$ foi de $1: 4,5$, a temperatura $156^{\circ} \mathrm{C}$ e o tempo 27 minutos. O hidrolisado foi três vezes concentrado sob vácuo a $75^{\circ} \mathrm{C}$ e sua composição foi determinada antes e depois deste processo como pode ser observado na Tabela 4.

Tabela 4. Composição química parcial do hidrolisado hemicelulósico de Eucalyptus grandis original e concentrado

\begin{tabular}{ccc}
\hline Componentes & $\begin{array}{c}\text { Hidrolisado } \\
\text { original (g/L) }\end{array}$ & $\begin{array}{c}\text { Hidrolisado } \\
\text { concentrado (g/L) }\end{array}$ \\
\hline Glicose & 2,54 & 6,50 \\
Xilose & 19,21 & 59,21 \\
Arabinose & 0,41 & 0,98 \\
Ácido acético & 5,03 & 6,33 \\
Fenóis & --- & 1,25 \\
\hline
\end{tabular}

Fonte: Canettieri; Silva e Felipe (2001).

$\mathrm{O} \mathrm{pH}$ do hidrolisado foi elevado de 1,9 para 7,0 com $\mathrm{CaO}$ e depois acidificado com $\mathrm{H}_{3} \mathrm{PO}_{4}$ até $\mathrm{pH}$ 5,5 . Em seguida foi submetido à adsorção com carvão ativo $10 \%$ sob agitação de $200 \mathrm{rpm}$, por 1 hora a $30^{\circ}$ C. Depois deste tratamento a quantidade de xilose era de $15 \mathrm{~g} / \mathrm{L}$ após a primeira concentração e $45 \mathrm{~g} / \mathrm{L}$ após a terceira concentração.

Para ser utilizado como meio de cultivo para produção de xilitol, o hidrolisado teve o $\mathrm{pH}$ e concentração de xilose ajustados e adição de sulfato de amônio e farelo de arroz de acordo com um modelo fatorial de $2^{4}$. Os coeficientes de regressão demonstraram que o $\mathrm{pH}$ e a concentração de xilose tiveram efeito significativo e positivo na produção de xilitol enquanto o sulfato de amônio teve efeito significativo porém negativo. Já o farelo de arroz não apresentou efeito significativo na produção do xilitol e por isso foi utilizado em pequena quantidade $(5 \mathrm{~g} / \mathrm{L})$.

O modelo matemático obtido previa uma produção de xilitol de 8,34 g/L utilizando hidrolisado contendo
$60 \mathrm{~g} / \mathrm{L}$ de xilose, suplementado com $1,1 \mathrm{~g} / \mathrm{L}$ de sulfato de amônio e pH inicial 8,0. Nestas condições de cultivo a produção de xilitol foi de $10,0 \mathrm{~g} / \mathrm{L}$, valor que está de acordo com o modelo previsto considerando o desvio padrão.

Mussato e Roberto (2002) utilizaram a palha de arroz como substrato na produção de xilitol. Para tanto a mesma foi submetida a um processo de hidrólise ácida $\left(\mathrm{H}_{2} \mathrm{SO}_{4}, 1 \% \mathrm{p} / \mathrm{v}\right)$ sob condições brandas $\left(120^{\circ} \mathrm{C}, 30\right.$ minutos). Em seguida o hidrolisado foi concentrado sob vácuo a $70^{\circ} \mathrm{C}$ com vistas a aumentar a concentração de xilose e tratado com carvão ativo em pó na proporção de $1 \mathrm{~g}$ de carvão: $40 \mathrm{~g}$ de hidrolisado em pH 2,0, agitação de $150 \mathrm{rpm}$, por 60 minutos a $45^{\circ} \mathrm{C}$. Este hidrolisado foi caracterizado físico-quimicamente em suas formas original, concentrado e tratado para determinação dos teores de glicose, xilose, arabinose, furfural, hidroximetilfurfural (HMF) e ácido acético e estimativa dos produtos de degradação de lignina (PDL) conforme pode ser observado na Tabela 5.

Tabela 5. Caracterização físico-química do hidrolisado hemicelulósico de palha de arroz original, concentrado e tratado com carvão ativo.

\begin{tabular}{cccc}
\hline Componentes & Original & Concentrado & Tratado \\
\hline Glicose & 3,29 & 19,80 & 18,75 \\
Xilose & 18,33 & 119,90 & 117,39 \\
Arabinose & 3,40 & 22,90 & 21,78 \\
Furfural & 0,10 & 0,07 & 0,00 \\
*HMF & 0,17 & 0,32 & 0,11 \\
Ácido acético & 1,05 & 2,28 & 1,97 \\
**PDL & 0,16 & 0,52 & 0,38 \\
pH & 1,22 & 0,40 & 0,40 \\
\hline
\end{tabular}

*Hidroximetilfurfural **Produtos de Degradação da Lignina Fonte: Mussato e Roberto (2002).

A análise da Tabela 5 mostra que o processo de hidrólise foi capaz de promover o fracionamento seletivo dos açúcares presentes na fração hemicelulósica da palha de arroz, liberando dentre estes, a xilose como principal constituinte. O processo fermentativo foi realizado com Candida 
guilliermondii durante $116 \mathrm{~h}$ tendo rendimento de $0,72 \mathrm{~g}$ de xilitol/ $\mathrm{g}$ de xilose e produtividade de 0,57 g/ L.h sem nenhuma suplementação do hidrolisado.

Herrera et al. (2004) avaliaram o efeito da concentração de ácido clorídrico na hidrólise da palha de sorgo sob pressão atmosférica. As hidrólises foram conduzidas a $100^{\circ} \mathrm{C}$ utilizando soluções de
$\mathrm{HCl}$ a 2, 4 ou $6 \%$ (p/v) e uma relação de $1 \mathrm{~g}$ de palha: 10 g de solução ácida. Após 20, 40, 60, 180 e 300 minutos foram retiradas amostras do meio reacional para determinação da composição físico-química do hidrolisado conforme pode ser observado na Tabela 6 onde o tempo de reação refere-se ao momento em que foi obtida a máxima concentração de xilose.

Tabela 6. Composição físico-química parcial do hidrolisado de palha de sorgo.

\begin{tabular}{cccccc}
\hline \multicolumn{7}{c}{ Composição (g/L) } \\
\hline$[\mathrm{HCl}](\mathrm{m} / \mathrm{v})$ & Xilose & Glicose & Furfural & Ácido acético & Tempo (min) \\
2 & 17,3 & 3,8 & 1,0 & 2,5 & 300 \\
4 & 19,9 & 5,1 & 1,1 & 3,6 & 300 \\
6 & 19,7 & 5,3 & 1,7 & 3,6 & 180 \\
\hline
\end{tabular}

Fonte: Herrera et al. (2004).

O modelo matemático obtido no estudo prevê máxima concentração de xilose (21,3 g/L) utilizando $\mathrm{HCl} \mathrm{6 \%}$ com tempo de hidrólise de 83 minutos.

Felipe et al. (2003) hidrolisaram casca de aveia utilizando relação de 1:4,5 para casca de aveia: solução de $\mathrm{H}_{2} \mathrm{SO}_{4} 0,35 \%$ temperatura de $156^{\circ} \mathrm{C}$ durante 27 minutos. O hidrolisado hemicelulósico foi tratado a partir do ajuste do $\mathrm{pH}$ inicial 2,22 para $\mathrm{pH}$ 7,0, com $\mathrm{CaO}$ comercial seguido do abaixamento para $\mathrm{pH} 2,5$ com $\mathrm{H}_{3} \mathrm{PO}_{4}$. Em seguida este foi submetido à adsorção em carvão ativado (1\%) em frascos agitados a $200 \mathrm{rpm}, 60^{\circ} \mathrm{C}$ por 30 minutos. A composição do hidrolisado após este tratamento pode ser observada na Tabela 7.

Tabela 7. Composição química parcial do hidrolisado hemicelulósico de casca de aveia após tratamento com $\mathrm{CaO}, \mathrm{H}_{3} \mathrm{PO}_{4}$ e carvão ativo.

\begin{tabular}{lc}
\hline \multicolumn{1}{c}{ Componentes } & Composição (g/L) \\
\hline Xilose & 32,33 \\
Glicose & 1,61 \\
Arabinose & 3,03 \\
Ácido acético & 3,63 \\
Furfural & 0,47 \\
Hidroximetilfurfural & 0,22 \\
\hline
\end{tabular}

A produção de xilitol por Candida guilliermondii neste hidrolisado suplementado com $\left(\mathrm{NH}_{4}\right)_{2} \mathrm{SO}_{4}$ $(2 \mathrm{~g} / \mathrm{L})$, solução de extrato de farelo de arroz (20 g/ $\mathrm{L})$ e $\mathrm{CaCl}_{2} \cdot 2 \mathrm{H}_{2} \mathrm{O}(0,1 \mathrm{~g} / \mathrm{L})$ teve rendimento de 0,54 $\mathrm{g} / \mathrm{g}$ de xilose e produtividade de $0,34 \mathrm{~g} / \mathrm{L}$. h após 46 horas de cultivo.

Comparação da produção de xilitol a partir de diferentes resíduos agroindustriais

Na Tabela 8 estão apresentadas as condições e hidrólise ácida e parâmetros fermentativos da produção de xilitol a partir dos diferentes resíduos agroindustriais apresentados anteriormente. Podem ser observadas grandes variações nestes parâmetros em função das condições de hidrólise, do tipo de tratamento aos quais foram submetidos os hidrolisados e dos diferentes microrganismos e condições de cultivo empregadas.

Fonte: Felipe et al. (2003). 
Tabela 8. Condições de hidrólise ácida e alguns parâmetros fermentativos da produção de xilitol utilizando diferentes resíduos agroindustriais

\begin{tabular}{|c|c|c|c|c|c|c|c|c|c|}
\hline Matéria prima & {$\left[\mathrm{H}_{2} \mathrm{SO}_{4}\right]$} & $\begin{array}{l}\text { Temp. } \\
\left({ }^{\circ} \mathrm{C}\right)\end{array}$ & $\mathbf{R} / \mathbf{A}$ & $\begin{array}{c}\text { Tempo } \\
\text { (minutos) }\end{array}$ & $\begin{array}{c}\text { [xilose] } \\
(\mathrm{g} / \mathrm{L})\end{array}$ & $\begin{array}{c}\text { [xilitol] } \\
\text { (g/L) }\end{array}$ & $\begin{array}{l}\text { Rend. em } \\
\text { produto } \\
(\mathrm{g} / \mathrm{g})\end{array}$ & $\begin{array}{c}\text { Prod. } \\
\text { Volumétrica } \\
\text { (g/L.h) }\end{array}$ & Referência \\
\hline Bagaço de cana & $100 \mathrm{mg} / \mathrm{g} \mathrm{ms}$ & 121 & $1: 10$ & 10 & 15,7 & - & - & 0,47 & Alves et al., 1998 \\
\hline Farelo de cevada & $3 \%(\mathrm{~m} / \mathrm{v})$ & 130 & $1: 8$ & 15 & 45,4 & 26,6 & 0,33 & - & Cruz et al., 2000 \\
\hline Sabugo de milho & $2 \%(\mathrm{~m} / \mathrm{v})$ & 130 & $1: 8$ & 15 & 42,9 & 29,0 & 0,26 & - & Cruz et al., 2000 \\
\hline Folhas de milho & $3 \%(\mathrm{~m} / \mathrm{v})$ & 130 & $1: 16$ & 15 & 48,1 & 21,2 & 0,36 & - & Cruz et al., 2000 \\
\hline Eucalyptus globulus & $3 \%(\mathrm{~m} / \mathrm{v})$ & 130 & $1: 8$ & 60 & 41,9 & 1,5 & 0,018 & - & Cruz et al., 2000 \\
\hline Eucalyptus grandis & $130 \mathrm{mg} / \mathrm{g} \mathrm{ms}$ & 156 & $1: 4,5$ & 27 & 19,21 & 10,0 & - & - & Canettieri; Silva; Felipe, 2001 \\
\hline Palha de trigo & $1 \%(\mathrm{~m} / \mathrm{v})$ & 140 & $1: 15$ & 20 & 15,33 & 27,86 & 0,889 & 0,503 & Canilha, 2001 \\
\hline Palha de arroz & $0,35 \%(\mathrm{~m} / \mathrm{v})$ & 120 & - & 30 & 18,33 & - & 0,72 & 0,57 & Mussato; Roberto, 2002 \\
\hline Casca de aveia & $0,35 \%(\mathrm{~m} / \mathrm{v})$ & 156 & $1: 4,5$ & 27 & 32,33 & - & 0,34 & 0,54 & Felipe et al., 2003 \\
\hline
\end{tabular}

$\left[\mathrm{H}_{2} \mathrm{SO}_{4}\right]=$ concentração de ácido sulfúrico

Temp $\left({ }^{\circ} \mathrm{C}\right)=$ temperatura ${ }^{\circ} \mathrm{C}$

[xilose $]=$ concentração de xilose $(\mathrm{g} / \mathrm{L})$

[xilitol $]=$ concentração de xilitol $(\mathrm{g} / \mathrm{l})$
Prod volumétrica $=$ produtividade volumétrica $(\mathrm{g} / \mathrm{L} . \mathrm{h})$

$\mathrm{mg} / \mathrm{g} \mathrm{ms}=\mathrm{mg}$ de $\mathrm{H}_{2} \mathrm{SO}_{4} / \mathrm{g}$ de matéria seca

Rend. em produto $=$ rendimento em produto $(\mathrm{g} / \mathrm{g})$

$\mathrm{R} / \mathrm{A}=$ relação massa seca $:$ solução ácida

\section{Recuperação do xilitol do meio fermentado}

A recuperação do xilitol é o passo mais complexo de todo o processo fermentativo devido à baixa concentração do produto e complexa composição do caldo fermentado. A literatura sobre recuperação de polióis de meios fermentados é bastante escassa (FAVERI et al., 2002).

Gurgel et al. (1995) desenvolveram uma metodologia para recuperação do xilitol produzido por fermentação do hidrolisado hemicelulósico de bagaço de cana-de-açúcar. A melhor condição de recuperação consistia na clarificação do meio com carvão ativado $(25 \mathrm{~g} / 100 \mathrm{~mL}$ de meio fermentado) a $80^{\circ} \mathrm{C}, \mathrm{pH} 6,0$ durante 60 minutos. O meio clarificado foi tratado com resinas de troca iônica, um procedimento que não se mostrou eficiente para purificação. Em seguida a solução foi concentrada e submetida à cristalização $\mathrm{a}-15^{\circ} \mathrm{C}$ monitorada por cinco semanas. Como durante este período não houve a formação de cristais visíveis, utilizou-se xilitol comercial na concentração de $1 \mathrm{~g} / \mathrm{L}$ como semente de cristalização. Após uma semana a $-15^{\circ} \mathrm{C}$ os cristais foram formados. Entretanto, apresentavamse envoltos em uma solução viscosa e rica em impurezas provenientes do caldo fermentado e que não foram removidas pelo tratamento com carvão ativado ou com resinas de troca iônica. Apesar disto, análises dos cristais mostraram serem estes bastante puros, com forma e cor uniformes.

Faveri et al. (2002) descrevem a recuperação do xilitol por cristalização de soluções de soluções sintéticas e de hidrolisados hemicelulósicos fermentados. O processo consistia na evaporação da solução a baixa pressão até super saturação, resfriamento da solução e separação dos cristais obtidos por centrifugação e filtração. Os melhores resultados, em termos de rendimento de cristalização $(0,56)$ ou grau de pureza $(1,00)$, foram obtidos em solução contendo $730 \mathrm{~g} / \mathrm{L}$ a uma temperatura de $-5^{\circ}$ C. Os autores concluíram que a recuperação do xilitol de hidrolisados hemicelulósicos fermentados por cristalização é um processo promissor.

\section{Considerações finais}

O interesse na obtenção e aplicações do xilitol têm aumentado nos últimos anos e como conseqüência tem-se procurado diminuir os custos de produção. O processo químico tradicionalmente utilizado em sua obtenção é bastante oneroso pois necessita de extensivas operações de purificação, as quais elevam custos e demandam tempo. O 
processo biotecnológico representa uma alternativa promissora, uma vez que utiliza microrganismos específicos para obtenção deste poliol e que não exigem a purificação requerida no processo químico. Em adição contribui com a melhoria das condições do meio ambiente pois são utilizados resíduos agroindustriais como fonte de xilose para a bioconversão deste produto.

\section{Referências}

ALVES, L. A.; FELIPE, M. G. A.; SILVA, J. B. E. S.; SILVA, S. S.; PRATA, A. M. R. Pretreatment of sugarcane bagasse hemicellulose hydrolysate for xylitol production by Candida guilliermondii. Applied Biochemistry and Biotechnology, Clifton, v.70-72, p.89-98, 1998.

ALVES, L. A Efeito do hidrolisado hemicelulósico de bagaço de cana-de-açúcar submetido a diferentes tratamentos sobre a atividade da xilose redutase de Candida guilliermondii. 2001. 193f. Tese (Doutorado) Faculdade de Ciências Farmacêuticas, Universidade de São Paulo (USP), São Paulo.

ALVES, L. A; VITOLO, M.; FELIPE, M. G. A.; SILVA, J.B. A. Xylose reductase and xylitol dehydrogenase activities of Candida guilliermondii as a function of different treatments of sugarcane bagasse hemicellulosic hydrolysate employng experimental design. Applied Biochemistry and Biotechnology, Clifton, v.98-100, p.403-413, 2002.

AGUIAR, C. L.; OETTERER; M.; MENEZES, T. J. B. Caracterização e aplicações do xilitol na indústria alimentícia. Boletim da Sociedade Brasileira de Ciência e Tecnologia e Alimentos, Campinas, v.33, n.2, p.184-193, $\mathrm{jul} / \mathrm{dez} 1999$.

AGUIAR JR, W. B.; FARIA, L. F. F.; COUTO, M. A. P. G.; ARAUJO, O. Q. F.; PEREIRA JR, N. P. Growth model and prediction of oxygen transfer rate for xylitol production from D-xylose by Candida guilliermondii. Biochemical Engineering Journal, Amsterdam, v.12, p.49-59, 2002.

AGUILAR, R.; RAMÍREZ, J. A.; GARROTE, G.; VÁZQUEZ, M. Kinetic study of the acid hydrolysis of sugar cane bagasse. Journal of Food Engineering, Essex, v.5, n.4, p.309-318, 2002.

BIRKHED, D. Cariologic aspects of xilitol and its use in chewing gum: a review. Acta odontologica Sscandinavica, Oslo, v.52, p.117-127, 1994.

BÔAS, S. G. V.; ESPOSITO, E. Bioconversão do bagaço de maçã. Biotecnologia Ciência e Desenvolvimento, Brasília, ano 3, n.14, p.38-42, maio/jun 2000.
CANILHA, L. Aproveitamento do hidrolisado de palha de trigo para produção de xilitol por via microbiana. 2001. Dissertação (Mestrado em Biotecnologia Industrial) - Faculdade de Engenharia Química de Lorena (FAENQUIL), Lorena.

CANILHA, L.; CÂNDIDO, E. J.; SILVA, J. B. A. Improvement in xylitol production achieved by the use of a wheat straw hemicellulosic hydrolisate. In: SEMINÁRIO DE HIDRÓLISE ENZIMÁTICA DE BIOMASSAS, 7., 2002, Maringá. Anais... Maringá: UEM, 2002. p.113.

CÂNDIDO, E. J.; CANILHA, L.; SILVA, J. B. A. Determinação da composição química da palha de trigo. In: SEMINÁRIO DE HIDRÓLISE ENZIMÁTICA DE BIOMASSAS, 7., 2002, Maringá. Anais... Maringá: UEM, 2002.p.108.

CANETTIERI, E. V.; SILVA, J. B. A. E.; FELIPE, M. G. A. Application of factorial design to the study of xylitol production from eucalyptus hemicellulosic hydrolysate. Applied Biochemistry and Biotechnology, Clifton, v.90, p.159-168, 2001.

CANETTIERI, E. V.; SILVA, J. B. A. E.; CARVALHO JR, J. A. Tratamento químico de resíduos de Eucalyptus grandis com ácido diluído. In: SIMPÓSIO NACIONAL DE FERMENTAÇÕES, 14., 2003, Florianópolis. Anais... Florianópolis, 2003. CDROM.

CRUZ, J. M.; DOMÍNGUEZ, J. M.; DOMÍNGUEZ, H.; PARAJÓ, J. C. Preparation of fermentation media from agricultural wastes and their bioconversion to xylitol. Food biotechnology, New York, v.14, p.79-97, 2000.

DOMÍNGUEZ, J. M.; CRUZ, J. M; ROCA, E.; DOMÍNGUEZ, H.; PARAJÓ, J. C. Xilytol production from wood hydrolyzates by entrapped Debaromyces hansenii and Candida guilliermondi. Applied Biochemistry and Biotechnology, Clifton, v.81, p.119-130, 1999.

ENDT, D. V.; COSTA, P.; ZAGO, M. K.; PASQUALI, G. Genes de lignificação. Biotecnologia Ciência e Desenvolvimento, Brasília, ano 3, n.15, p.152-159, jul/ago 2000. Encarte Especial.

FELIPE, M. G. A.; HAULY, M. C. O.; CANETTIERI, E. V; CÂNDIDO, E. J; TAMANINI, C. Avaliação da casca de aveia para obtenção de hidrolisado hemicelulósico e produção de xilitol por processo fermentativo. In: SIMPÓSIO NACIONAL DE FERMENTAÇÕES, 14., 2003, Florianópolis. Anais... Florianópolis, 2003. CDROM.

FAVERI, D.; PEREGO, P.; CONVERTI, A.; BORGHI, M. Xylitol recovery by crystallization from synthetic solutions and fermented hemicellulose hydrolyzates. Chemical Engineering Journal, Lausanne, v.90, p.291-298, 2002.

FAVERI, D.; TORRE, P.; PEREGO, P., CONVERTI, A. Optimization of xylitol recovery by cristallization from 
synthetic solutions using response surface methodology. Journal of Food Engineering, Essex, 2003 (in press).

FERRAZ, A. Aplicações da biotecnologia na produção de papel e celulose. In: LIMA, U. A. (Coord.). Biotecnologia Industrial. São Paulo: Edgar Blücher, 2001. v.3, p.465-484.

GALDEANO, M. C. Caracterização e aplicação de casca de aveia modificada por tratamento com peróxido de hidrogênio alcalino associado à extrusão. 2001. Dissertação (Mestrado em Ciência e Tecnologia de Alimentos) - Departamento de Tecnologia de Alimentos e Medicamentos, Universidade Estadual de Londrina, Londrina.

GARCIA, A. D.; PRATA, A. M. R. Utilização do hidrolisado hemicelulósico de eucalipto para produção de 2,3 butanodiol pela bactéria Klebsiella pneumoniae. In: REUNIÃO NACIONAL DE MICROBIOLOGIA APLICADA AO MEIO AMBIENTE, 2. ,1998, Taubaté. Anais... Taubaté, 1998. CDROM.

GURGEL, P. V.; MANCILHA, I. M.; PEÇANHA, R. P.; SIQUEIRA, J. F. M. Xylitol recovery from fermented sugarcane bagasse hydrolyzate. Bioresource Technology, Essex, v.52, p.219-223, 1995.

HERRERA, A.; SIMÓN, J. T. L.; CABRIALES, J. J. G.; RAMÍREZ, J. A.; VÁZQUEZ, M. Effect of the hydrochloric acid concentration on the hydrolysis of sorghum straw at atmospheric pressure. Journal of Food Engineering, Essex, v.63, n.1, p.103-109, 2004.

HERRERA, A.; TÉLLEZ-LUIS, S. J.; RAMÍREZ, J. A.; VÁZQUEZ, M. Production of xylose from sorghum straw using hydrochloric acid. Journal of Cereal Science, London, v.37, p.267-274, 2001.

LIMA, L. H. A.; BERLINCK, C. N. Xilitol: o adoçante do futuro. Ciência Hoje, São Paulo, v.33, n.195, p.6669, jul./2003.

MARTON, J. M. Avaliação de diferentes carvões ativos $e$ das condições de adsorção no tratamento do hidrolisado hemicelulósico de bagaço de cana para obtenção biotecnológica de xilitol. 2002. Dissertação (Mestrado em Biotecnologia) - Departamento de Biotecnologia, Faculdade de Engenharia Química de Lorena, Lorena.

MORITA, T. A.; SILVA, S. S.; MAUGERI, F. Estudo de processo integrado de produção de xilitol por fermentação. In: SIMPÓSIO NACIONAL DE FERMENTAÇÕES, 14., 2003, Florianópolis. Anais... Florianópolis, 2003. CD-ROM.

MUSSATO, I. S.; ROBERTO, I. C. Produção biotecnológica de xilitol a partir da palha de arroz. Biotecnologia Ciência e Desenvolvimento, Brasília, n.28, p.34-39, set/out 2002.
NEUREITER, M.; DANNER, H.; THOMASSER, C.; SAIDI, B.; BRAUN, R. Dilute-acid hydrolysis of sugarcane bagasse at varying conditions. Applied Biochemistry and Biotechnology, Clifton, v.98-100, p.49-58, 2002.

OLIVEIRA, R. M.; CARVALHO, E. P.; SCHUWAN, R. F. Produção de enzimas hidrolíticas extracelulares por Fusarium em sistema de batelada simples. Revista Interação, Juiz de Fora, n.2, 2000. Disponível em: $<$ http://interacao.unis.edu.br/ n2/art2.htm>. Acesso em: 14 out. 2003.

PALMQVIST, E.; HAHN-HÄGERDAL, B. Fermentation of lignocellulosic hydrolysates. I: inhibition and detoxification. Bioresource Technology, Essex, v.74, p.17$24,2000$.

PARAJÓ, J. C.; DOMÍNGUEZ, H.; DOMÍNGUEZ, J. M. Biotechnological production of xylitol. Part 2: operation in culture media made with commercial sugars. Bioresource Technology, Essex, v.65, p.203-212, 1998a.

PARAJÓ, J. C.; DOMÍNGUEZ, H.; DOMÍNGUEZ, J. M. Biotechnological production of xylitol. Part 2: operation in culture media from lignocellulose hydrolysates. Bioresource Technology, Essex, v.66, p.25-40, 1998b.

ROBERTO, I. C.; FELIPE, M. G. A.; MANCILHA, I. M.; VITOLO, M.; SATO, S.; SILVA, S. S. Xylitol production by Candida guilliermondii as an approach for the utilization of agroindustrial residues. Bioresource Technology, Essex, n.51, p.255-257, 1995.

ROBERTO, I. C.; MANCILHA, I. M.; SATO, S. Influence of $\mathrm{k}_{\mathrm{L}} \mathrm{a}$ on bioconversion of rice straw hemicellulose hydrolysate to xylitol. Engineering, Knowle, n.21, p.505508, 1999.

RODRIGUES, R. C. L. B.; FELIPE, M. G. A.; SILVA, J. B. A; VITOLO, M.; GÓMEZ, P. V. The influence of $\mathrm{pH}$, temperature and hydrolysate concentration on the removal of volatile and nonvolatile compounds from sugarcane bagasse treated with actived charcoal before or after vacuum evaporation. Brazilian Journal of Chemical Engineering, São Paulo, v.18, n.3, p.299-311, 2001.

RODRIGUES, R. C. L. B.; FELIPE, M. G. A.; PESSOA JR, A.; PRATA, A. M. R. Crescimento de Candida guilliermondii durante cultivo descontínuo em hidrolisado hemicelulósico de bagaço de cana: efeito do O. In: SIMPÓSIO NACIONAL DE FERMENTAÇÕES, 14., 2003, Florianópolis. Anais... Florianópolis, 2003a. CDROM.

RODRIGUES, R. C. L. B.; FELIPE, M. G. A.; SILVA, J. B. A; VITOLO, M. Response surface methodology for xylitol production from sugarcane bagasse hemicellulosic hydrolysate using controlled vacuum evaporation process variables. Process Biochemistry, Essex , v.38, p.1231-1237, $2003 b$. 
SAHA, B. C. Hemicellulose bioconversion. Journal of Industrial Microbiology and Biotechnology, Hampshire, v.30, p.271-291, 2003.

SHREVE, R. N.; BRINCK JR, J. A. Derivados químicos da madeira. In: Indústrias de processos químicos. Rio de Janeiro: Guanabara Koogan, 1977. p.487-495.

SOUZA, O.; SANTOS, I. E. Importância dos resíduos agropecuários na alimentação animal. Boletim pecuário. 2002? Disponível em: <http:// www.boletimpecuario.com.br/artigos/ showartigo.php?arquivo=artigo544.txt $>$. Acesso em: 11 out. 2003.
SUN, Y.; CHENG, J. Hydrolysis of lignocellulosic materials for ethanol production: a review. Bioresource Technology, Essex, v.83, p.1-11, 2002.

VERDE, M. V. Aplicação de resinas de troca iônica no tratamento de hidrolisado hemicelulósico para processos fermentativos. 2001. Dissertação (Mestrado) Departamento de Biotecnologia, Faculdade de Engenharia Química de Lorena, Lorena.

WINKELHAUSEN, E.; KUZMANOVA, S. Microbial conversion of D-xylose to xylitol. Journal of Fermentation and Bioengineering, Osaka, v.86, n.1, p.1-14, 1998. 
